Estudios sobre armas antiguas, armamento, arte militar $\mathrm{y}$ vida cultural en oriente y occidente

XXVI (2006), pp. 93-104

ISSN: 0435-029X

\title{
FALCATA IBÉRICA CON DECORACIÓN DAMASQUINADA PROCEDENTE DEL YACIMIENTO DE CUESTA DEL ESPINO (CÓRDOBA $)^{1}$
}

\author{
POR \\ Manuel Sierra Montesinos* \\ ANTONiO MARTíneZ CASTRO*
}

\section{RESUMEN - ABSTRACT}

Presentamos un ejemplar de falcata aparecido de forma casual, que pese a estar incompleta resulta muy interesante por estar decorado y por conocer con bastante seguridad su lugar de procedencia, del cual se realiza una aproximación a su contexto arqueológico, creemos funerario, en que debe insertarse dicha pieza, así como un primer estudio de sus características formales y decorativas.

We would like to introduce an example of falcata, found by chance, which, in spite of not being completed at all, becomes very interesting due to its ornaments and also, due to know where it comes from for sure, from which we can deduce an arqueological approximation to the context, we believe funereal in which we must settle the piece down, as well as a first report on its formal and ornamental features.

\section{PALABRAS CLAVE - KeYWORDS}

Cultura ibérica, arqueología ibérica, armamento ibérico, falcata.

Iberian Culture, Iberian Arqueology, Iberian weapons, falcata.

\section{Circunstancias DEL HALlAZGO}

La pieza que estudiamos fue hallada por un vecino de La Carlota, que la entregó al Ayuntamiento de La Carlota, (donde se conserva en la Colección Arqueológica Municipal de dicho municipio), en un lugar situado al borde de la Autovía de Andalucía (antigua Nacional IV Madrid-Cádiz), donde, por parte de la Administración Pública responsable de las obras ligadas a la mencionada arteria de comunicación, se han llevado a cabo movimientos de tierra para el acondicionamiento de los márgenes de la Autovía con motivo de la habilitación del tercer carril en sentido Madrid-Cádiz al coronar la llamada «Cuesta del Espino».

$\mathrm{Al}$ efectuarse dicha remoción se produjo el afloramiento de la pieza y también de una serie de manchas oscuras que destacaban en el terreno grisáceo-amarillento mioceno y que

\footnotetext{
* Universidad de Córdoba.

1 El presente trabajo se inscribe dentro de una de las líneas de investigación del Proyecto de Investigación desarrollado por el Grupo de Investigación HUM 721 del Plan Andaluz de Investigación.
} 
denotaban un posible origen arqueológico. Comprobando visualmente las características de dichas manchas se llegó a la conclusión no sólo de que, efectivamente, eran arqueológicas sino también de que se estaba claramente ante un contexto funerario ibérico, pues en ellas se observaban fragmentos de cerámicas pintadas -presumiblemente urnas-, restos de hierro así como trozos de huesos procedentes de cremaciones. El hecho de que nuestra falcata aparezca doblada sobre sí es prueba también de esa vinculación con el mundo de ultratumba.

\section{ConTeXto ARQueOlógico: EL YACIMIENTO IBÉRICO DE CUESTA DEL ESPINO}

(Láms. 1, 2, 3) (Figs. 1, 2).

Son inexistentes a la investigación las necrópolis ibéricas localizadas hasta ahora en la zona que tratamos, y escasos los asentamientos correspondientes a la misma etapa. Aunque el yacimiento ibérico de Cuesta del Espino era conocido ya por la investigación, se desconocía totalmente la ubicación de su posible necrópolis. Si tenemos en cuenta que el lugar de hallazgo de nuestra pieza está situado a menos de medio kilómetro en línea recta de dicho asentamiento, podemos pensar con cierta posibilidad que se trata del cementerio a él asociado, puesto que en un radio de varios kilómetros -al menos una decena- no se conoce otro asentamiento de similares características y envergadura. La ubicación es por otro lado, perfecta, en la ladera de un cerro separado del asentamiento sólo por una vaguada.

Del posible oppidum de Cuesta del Espino ya se ha publicado por uno de nosotros,algún material de la misma época, concretamente un conjunto de tres braserillos de bronce hallados en el cerro donde se emplaza el asentamiento. Fueron hallados de forma casual y resultan de gran interés por la escasez de este tipo de piezas no sólo en esta zona, sino también en la provincia cordobesa, y sobre todo porque aportan nuevos datos relativos a la tipología y fabricación de estos recipientes ${ }^{2}$. Igualmente, de suma importancia es el hecho de que nos revelen que al menos en su contexto geográfico próximo estamos ante un yacimiento muy interesante, capaz de proporcionar materiales de la calidad y relevancia de los mencionados braserillos o la pieza que aquí presentamos.

El yacimiento de Cuesta del Espino fue publicado en un estudio sobre el poblamiento protohistórico en el sureste de la Provincia de Córdoba dentro de un proyecto más amplio de investigación sobre la Subbética cordobesa llevado a cabo por la Universidad de Córdoba en los últimos años de la década de $1980^{3}$. Según este estudio, Cuesta del Espino es clasificado como un asentamiento significativo a la hora de analizar la estructura del poblamiento ibérico del cuadrante suroriental de la provincia de Córdoba, limitando su territorio teórico con los importantes núcleos de Corduba, Ulia y Ategua.

A tenor de los datos observados, creemos que el yacimiento ibérico de Cuesta del Espino pudo ser, efectivamente, un oppidum, aunque su tamaño está todavía por determinar, siendo necesaria para aclarar este dato la realización futura de estudios más profundos. Asimismo, es difícil hacerse una idea completa de la morfología y características de este yacimiento, pues se halla muy alterado por las constantes obras públicas destinadas al trazado de las sucesivas carreteras, ya que siempre ha sido éste un lugar obligado de paso para las comunicaciones entre la Meseta y la Baja Andalucía al menos desde la época republicana

2 Martínez Castro, A.; Tristell Muñoz, F. J., «Nuevos aspectos sobre los llamados 'braserillos' ibéricos a la luz de un reciente descubrimiento. El conjunto de Cuesta del Espino (Córdoba)», Antiqvitas, 11-12 (2000), pp. 19-26.

3 Murillo, J. F. et alii, «Aproximación al estudio del poblamiento protohistórico en el sureste de Córdoba: unidades políticas, control del territorio y fronteras», Fronteras. Arqueología Espacial, 13 (1989), pp. 151-172.

4 Ruiz, A.; Molinos, M., Los iberos. Análisis arqueológico de un proceso histórico, Barcelona, 1993, pp.116 y 141 . 


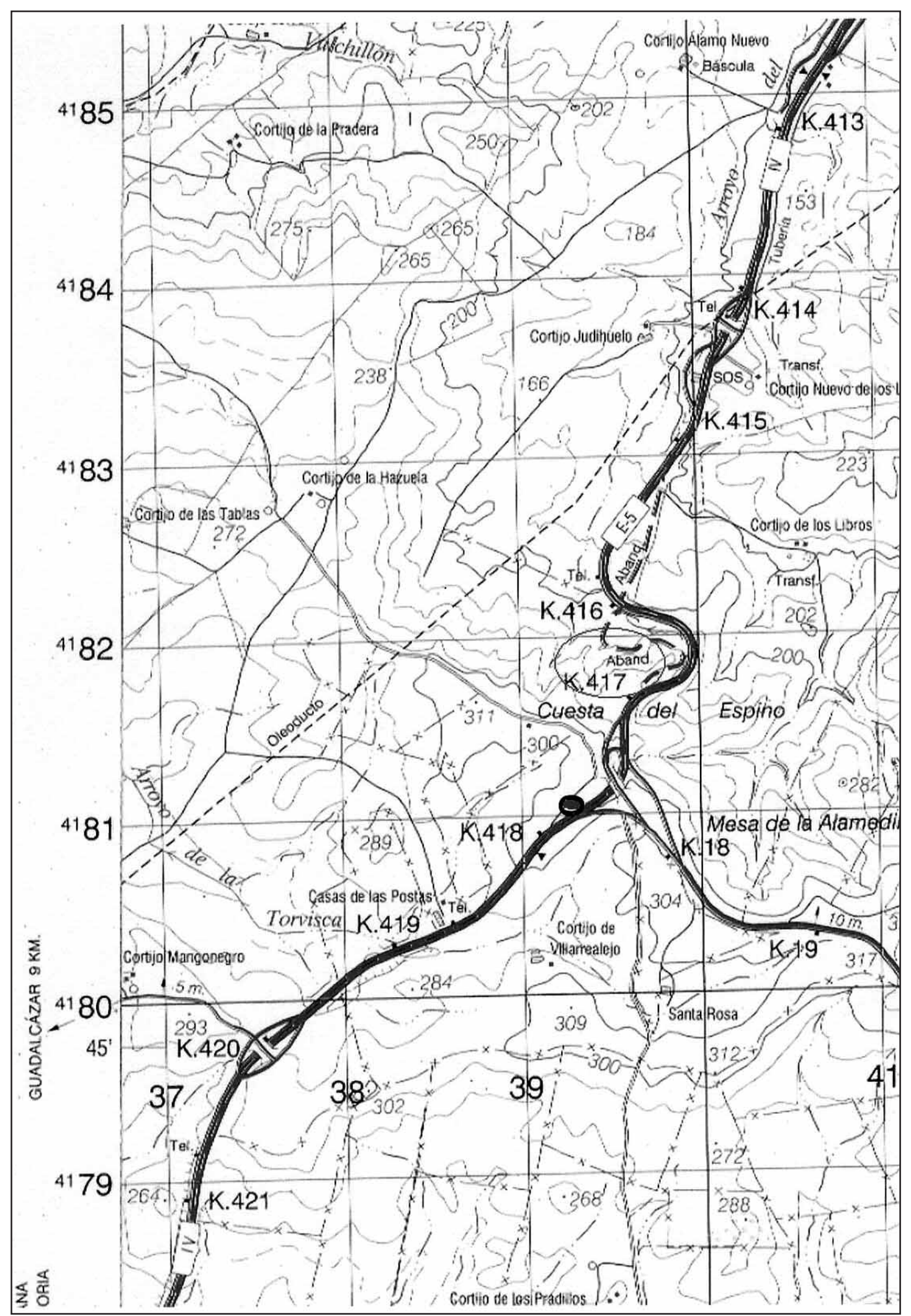

Fig. 1. Situación del hallazgo (elipse pequeña) en relación al yacimiento (elipse grande). Hoja n. ${ }^{\circ} 944$ del Mapa Militar de España. Serie L (Escala 1:50.000. 


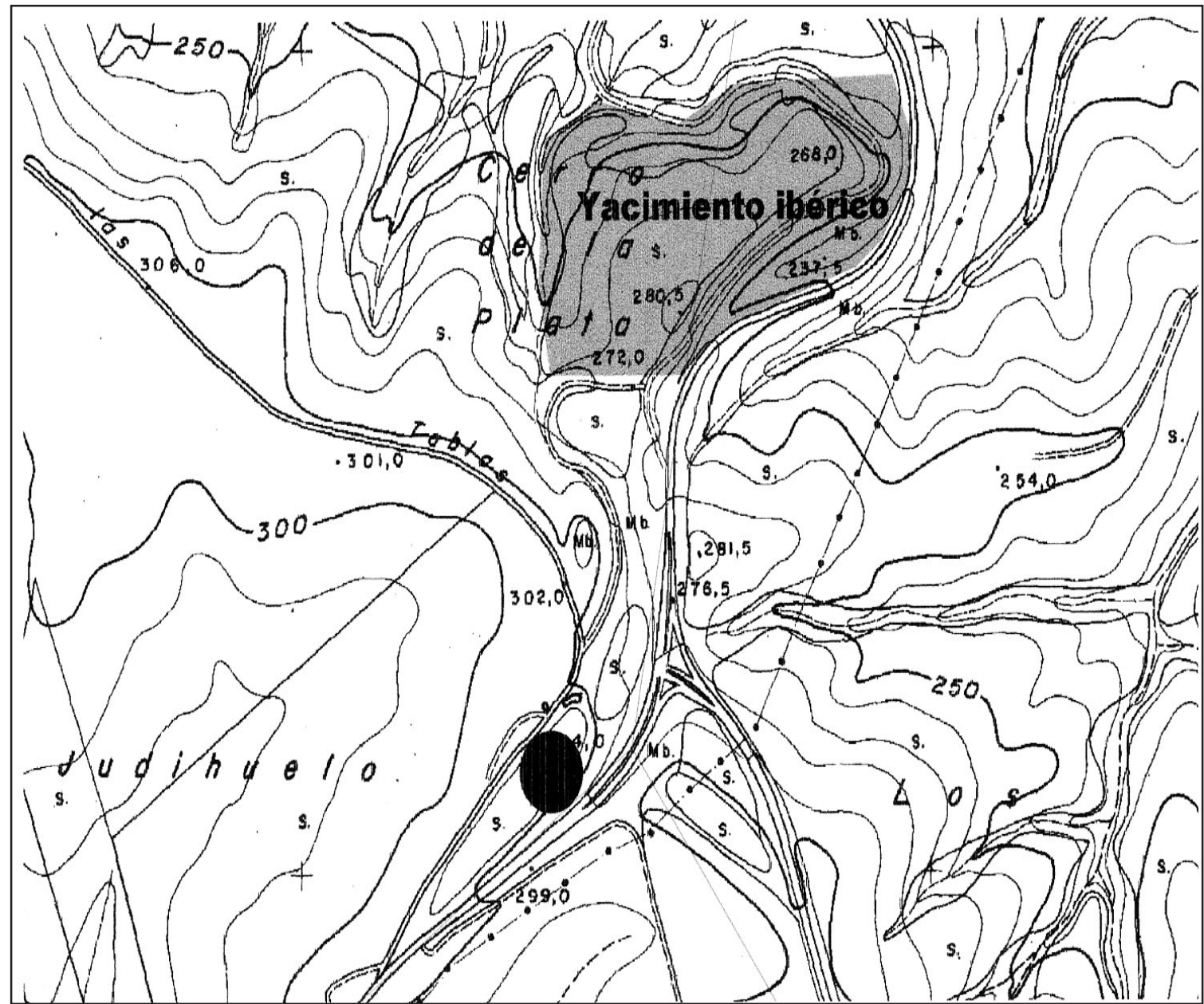

Fig. 2. Lugar del hallazgo en M.T.A. 1:10.000 Hoja D.94412. Unos 485 m. de distancia entre éste y el yacimiento ibérico.

romana $^{5}$. Lo que sí está más claro es que el de Cuesta del Espino era un asentamiento que controlaba un punto geográfico estratégico ${ }^{6}$, como consecuencia de la litología miocena y de su situación geográfica, pues se sitúa sobre un cerro-testigo al borde del curso bajo

5 Para la época romana vid.: Melchor Gil, E., «Comunicaciones entre Astigi y la Campiña de Córdoba en época romana: Via Augusta y Camino de Metedores», Ariadna 8 (1990), pp. 71-97; Melchor GIL, E., Vias romanas de la provincia de Córdoba, Córdoba 1995 y CoRzo, R.; TosCANo, M., Las vías romanas de Andalucía, Sevi1la, 1992.Además el investigador francés Pierre Sillières recoge un testimonio indicativo de que ya en época romana el lugar sufrió los efectos de una obra viaria, ya que este investigador halló en Cuesta del Espino restos de la caja que alojó a la Via Augusta (Sillières, P., «La Via Augusta de Cordoue a Cadix», Mélanges de la Casa de Valazquez, 12 (1976), p 49.Los sucesivos trazados de las carreteras entre Córdoba y Sevilla también se han efectuado por este lugar: el arrecife árabe y moderno, el Camino de Andalucía del siglo XVIII, la carretera de primer orden del Circuito Nacional de Firmes Especiales primorriverista, la carretera Radial-nacional IV del franquismo y, actualmente la Autovía de Andalucía, construída a inicios de la década de 1990.Para comprobar cómo todos estos trazados de la carretera entre Córdoba y Sevilla han pasado por la Cuesta del Espino basta con consultar AA.VV., El Camino de Andalucía. Itinerarios Históricos entre la Meseta y el Valle del Guadalquivir,Madrid, 1993). Todos los trazados han debido de afectar, al menos parcialmente, al yacimiento ibérico sobre el que tratamos, como aún se advierte directamente y a simple vista en el lugar.

${ }^{6}$ La zona en cuestión se halla a una altitud aproximada de $300 \mathrm{~m}$ sobre el nivel del mar. Se trata de un punto 
del Guadajoz. Además, el punto constituía -y aún hoy lo sigue haciendo- un auténtico nudo de comunicaciones de primer orden, ya que en él se cruzan la vía que conectaba la Meseta con el Valle del Guadalquivir y la costa atlántica española (antigua Via Augusta romana) y la vía que comunicaba el valle medio del Guadalquivir con la costa sur mediterránea (vía Corduba-Anticaria-Malaca romana), vías posiblemente ya existentes desde época prerromana ${ }^{7}$.

Respecto a las características cronológicas y culturales del asentamiento, sólo podemos atisbar algunos rasgos generales si nos atenemos a los materiales con que contamos. En primer lugar, las cerámicas halladas en el lugar ${ }^{8}$ nos indican que este yacimiento es ocupado en la etapa del Bronce Final -Orientalizante, según se desprende de la presencia de bruñidas de carena alta, grises, pintadas polícromas, etc., lo que nos sitúa entre los siglos VIII-VI a. C. También abundan las cerámicas ibéricas, cuya cronología a primera vista y en virtud de las piezas con que contamos no podemos precisar por falta de indicadores claros, cuestión que exigiría una rigurosa investigación arqueológica (con prospección y excavación), así como un análisis profundo de los materiales obtenidos, aunque sí sabemos que la Baja Época ibérica está representada, pues contamos con la presencia de cerámicas campanienses. A tenor de estos datos nos inclinamos a pensar que el pleno iberismo también se hallaría representado en este asentamiento, debiendo existir cierta continuidad entre la fase orientalizante y el bajo iberismo.

\section{LA FALCATA. DESCRIPCIÓN Y CARACTERÍSTICAS FORMALES} (Láms. 4, 5, 6, 7) (Fig. 3)

La pieza no se conserva entera, faltándole el tercio distal y buena parte del tercio medial de la hoja. Seguimos los criterios de descripción propuestos por el profesor F. Quesada (QuESADA, 1992:89; 1997:84).

Conserva buena parte de la empuñadura aunque le falta el extremo de ésta, no siendo posible determinar si se trataba de una pieza con cabeza de ave o de caballo, correspondiendo al tipo C de Quesada (QuesadA, 1997:102). Tampoco es posible determinar si tenía o no guarda lateral, de cadenilla o maciza. Sí se aprecian dos remaches redondeados para fijación de las cachas de material orgánico que se han perdido. Presenta oxidación superficial con adherencia de materiales terrosos. La corrosión ha hecho mella en la superficie original que ha caído en forma de láminas que han afectado especialmente a las partes frontales de la

privilegiado en el entorno, al situarse en la primera y más alta terraza de las cinco localizadas en el valle del Guadalquivir cordobés (terrazas Cuesta del Espino-Malpartida, entre 255 y $155 \mathrm{~m}$ sobre el nivel del río).Vid. LÓPEZ Ontiveros, A., «Rasgos geomorfológicos de la Campiña de Córdoba», Estudios Geográficos, 130 (febrero 1973), p. 52.

7 Pierre Sillières ha señalado la existencia de la Via Augusta al menos desde épòca anterior a Augusto (SILLIÈRES, P., «Les sources littéraires et le réseau routier de l'Hispanie Méridionale a l'époque républicaine», en GonZÁlez, J. (ed.), Estudios sobre Urso. Colonia Iulia Genetiva, Sevilla, 1989, p. 362). También el profesor Enrique Melchor, siguiendo a este autor, indica que su origen es anterior al Imperio, habiendo sido construida por el ejército romano, según se deduce de su denominación como Via augusta Militaris que recibe en un miliario hallado cerca de Córdoba y estudiado por el mismo P. Silliéres (vid. Melchor Gil, E., Vias romanas de la provincia de Córdoba, Córdoba, 1995, p. 97 y Silliéres, P., «A propos d'un nouveau milliaire de la Via Augusta, une Via Militaris en Bétique», Revue des Études Anciennes, 83 (1981), pp. 262-271). Respecto a la vía Corduba-Malaca, E. Melchor piensa, siguiendo los estudios de P. Rouillard, que es también prerromano, pues este itinerario muestra una vía de penetración de productos cerámicos griegos desde la costa hacia Corduba. Igualmente, el tramo de esta vía entre Corduba y Ulia tuvo que ser usado por Cneo Pompeyo tras levantar el sitio de Ulia (MelCHOR GIL, E., op cit., pp. 112-113). doba).

8 Estas cerámicas se hallan depositadas en el Museo Histórico Local «Juan Bernier» de La Carlota (Cór- 

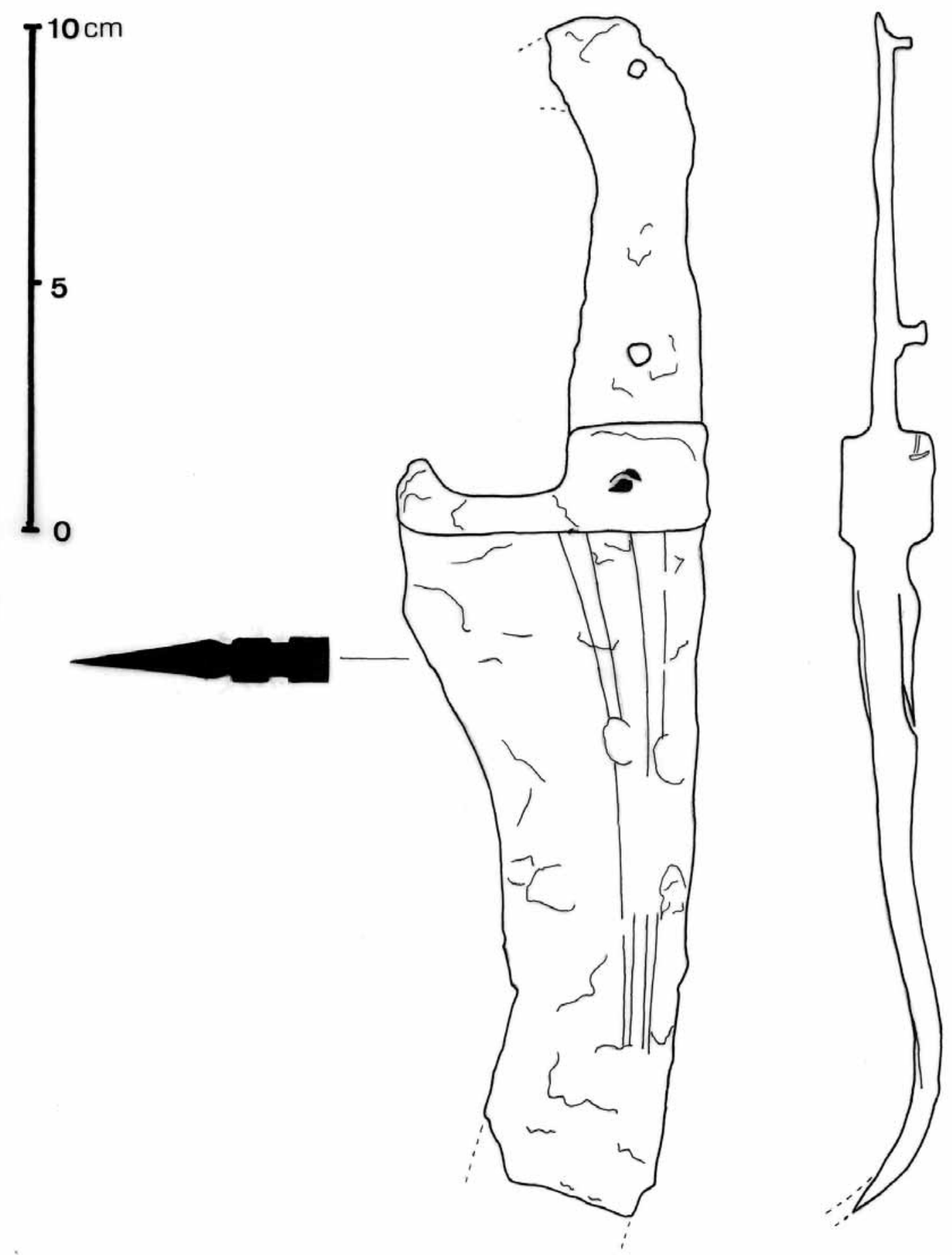

Fig. 3. Falcata con decoración. 
guarda basal, lugar donde hemos detectado decoración damasquinada en plata. El extremo conservado de la hoja termina en forma sinuosa, creemos que por efecto de la acción intencionada al inutilizar el arma, que ha hecho perder el resto de la hoja. En ésta presenta dos acanaladuras divergentes que parten de la base, la más próxima casi paralela al dorso, de base plana, y la más alejada y menos pronunciada de sección en plano inclinado al arranque de la hoja, que va estrechándose, tendiéndose a unir en su avance por la hoja con la primera acanaladura, rasgo muy frecuente en las piezas de la necrópolis de Los Collados de Almedinilla (QUESADA, 1997: LÁM. II C). Por la disposición de las acanaladuras en la parte más próxima a la empuñadura se trata de un tipo 1 (QUESADA, 1997: 94). No se aprecian señales de mellado intencional del filo.

Las dimensiones del arma son las siguientes: Longitud máxima (conservada): 24 '4 cm. Longitud de la hoja (conservada):14 cm. Longitud de la empuñadura (conservada): $10^{\prime} 4 \mathrm{~cm}$. Longitud del filo dorsal: no se aprecia en la parte de la hoja conservada. Anchura máxima de la hoja (conservada) en el arranque de la base: $6 \mathrm{~cm}$. Anchura mínima de la hoja (conservada): 2'9 cm. Grosor máximo de la hoja (conservada): 1'1 cm. Si tratásemos de reconstruir la longitud total de la hoja, por supuesto de modo especulativo, en función del fragmento que le falta, y de que el doblez de la hoja tuvo que encontrarse cerca del punto de equilibrio del arma, diríamos que alcanzaba unos $42 \mathrm{~cm}$. Con un márgen de varios centímetros abajo o más bien arriba de ésta cifra. Sin embargo, pensamos que las dimensiones totales de la falcata la acercan al grupo de las falcatas andaluzas (QuESADA, 1992:56).A pesar de todo ello, el estado de conservación lo hemos de calificar como de malo.

La guarda basal la identificamos con un tipo 2B de la tipología de E. Cuadrado (CuADRADO, 1989: 18-20).

En lo concerniente a la decoración, la pieza presenta en uno de los frontales de la guarda basal decoración damasquinada en plata consistente en dos hojas de hiedra ${ }^{9}$. Además en la parte dorsal de la guarda se observan a simple vista dos hilillos de plata que denotan la presencia de decoración damasquinada debajo de la capa de óxido superficial ${ }^{10}$.

A pesar de representar quizá una pequeña parte de la decoración total de la pieza, el motivo decorativo de las hojas de hiedra está bien representado en el repertorio decorativo de las falcatas ${ }^{11}$ relacionado con el símbolo de la inmortalidad (QUESADA, 1997:117).

Los paralelos más claros, también los más cercanos, creemos encontrarlos en la zona de Almedinilla -Illora, por la repetición del motivo decorativo de las hojas de hiedra y también por el tipo y disposición de las acanaladuras relacionadas con las falcatas cordobesas, como más arriba hemos expuesto.

\section{CONCLUSIONES}

Pese a su lamentable estado de conservación la falcata del Cerro del Espino posee cierta singularidad. Se trata, según nuestras noticias, de la pieza decorada más occidental de Andalucía y una de las mas occidentales de la Península Ibérica (fig. 4), por lo que creemos que puede ayudar en los estudios de dispersión de estas bellas muestras de la metalistería ibérica.

9 Llegamos a tal constatación al desprenderse una lámina de óxido de la cartela de la guarda basal cuando manipulábamos la pieza para obtener sus medidas y observamos una hoja de hiedra junto a otra mayor, en sentido contrario (lám.7).

10 Sometimos la pieza a una serie de sesiones radiográficas, para intentar revelar la decoración de las zonas que suponíamos que no había saltado, sin conseguir penetrar en el núcleo metálico de la guarda basal.

11 Ver cuadro de los motivos decorativos de las falcatas en QuesadA SANZ, F., El armamento ibérico. Estudio tipológico, geográfico, funcional, social y simbólico de las armas en la Cultura Ibérica (siglos VI- I a. C.).Monographies Instrumentum 3. Montagnac, 1997, pp.121, números 11al 16. 


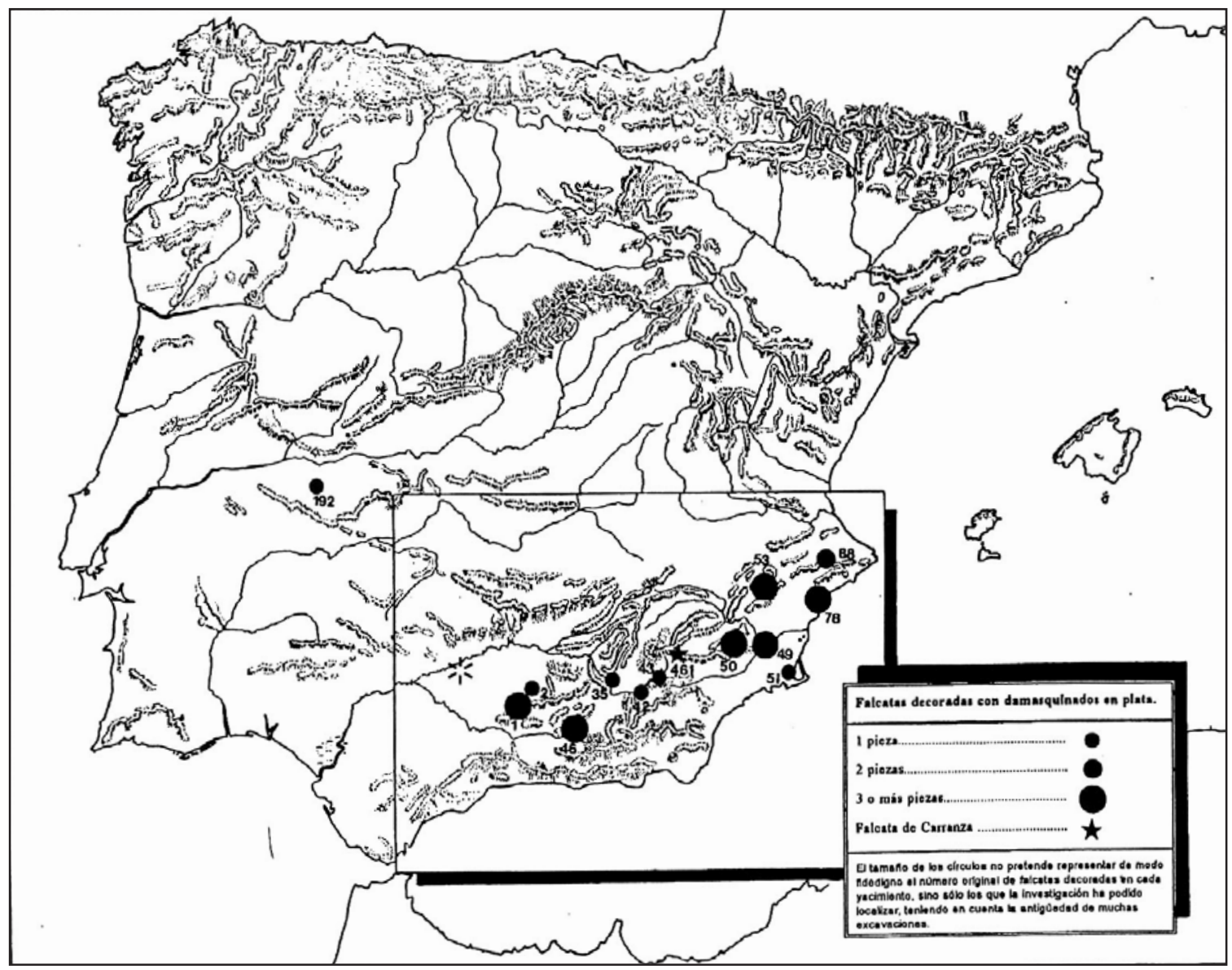

Fig. 4. Distribución de las falcatas con decoración damasquinada.

Tomado de Quesada (QuesADA,1997:11.5),con inclusión de nuestro hallazgo (sin numeración).

Por otro lado, debemos resaltar el contexto, a nuestro juicio de carácter funerario en el que se encontró la pieza, aunque los indicios presentados más arriba no sean absolutamente determinantes, siendo deseable algún tipo de intervención que pudiera esclarecer éste y otros problemas aquí planteados.

Pensemos además, que la relación de nuestra falcata con la zona de Almedinilla se materializa en el motivo decorativo y en la disposición y forma de las acanaladuras conservadas, aunque el modo en que actuó dicha influencia se nos escape ${ }^{12}$ con tan sólo dos hojas de hiedra y un par de hilillos en el dorso de la guarda basal. Por ello también resulta muy difícil tratar el aspecto cronológico. Si mantenemos las relaciones con Almedinilla y en esta zona tenemos una cronología que abarca del siglo V-II a. C.,podríamos suponer con las debidas reservas similar cronología, que tampoco aclara demasiado pero que encaja holgadamente en el desarrollo cultural, bosquejado más arriba, del opidum del Cerro del Espino.

12 Recientemente (Quesada Sanz, F.; Gabaldón Martínez, M.; RequenA, F.; Zamora, M, «¿Artesanos itinerantes en el mundo ibérico? Sobre técnicas y estilos decorativos, especialistas y territorio» III Reunió sobre Economía en el Mon Ibèric. Saguntum-PLAV, Extra 3, pp. 291-301.) se ha formulado la hipótesis de artesanos itinerantes que recorrían el espacio ibérico ofreciendo sus servicios y que serían los responsables del llamado «estilo libre» frente al «estilo preciso o minucioso». Con la escasa decoración damasquinada conservada susceptible de ser observada es imposible adscribirse a uno u otro estilo por atractiva que resulte tal hipótesis. 


\section{BIBLIOGRAFÍA}

AA.VV. (1993): El Camino de Andalucía.Itinerarios históricos entre la Meseta y el Valle del Guadalquivir.Madrid.

Cortio Cerezo, M. ${ }^{a}$ L. (1990): El municipio romano de Ulia (Montemayor, Córdoba).Córdoba.

Corzo Sánchez, R.; Toscano San Gil, M. (1992): Las vías romanas de Andalucía. Sevilla.

CuAdrado Díaz, E. (1987): La necrópolis ibérica de «El Cigarralejo»(Mula, Murcia). B.P.H. XXIII. Madrid.

Cuadrado DíAz, E. (1989) La panoplia ibérica de El Cigarralejo (Mula, Murcia).Serie Arqueología, n. ${ }^{\circ}$ 3. Murcia.

LACORT NAVARRO, P. J. (1985): «Cereales en Hispania Ulterior:Silos de época ibérica e ibero-romana en la Campiña de Córdoba»,,Habis 16, pp. 363-386.

López Ontiveros, A. (1973): «Rasgos geomorfológicos de la Campiña de Córdoba».Estudios Geográficos, 130, pp. 31-94.

Martínez Castro, A.; Tristell MuÑoz, F. J. (1999): «Notas sobre carámica ibérica procedentes del noroeste de la Campiña de Córdoba (Términos municipales de La Carlota y Córdoba)» Antiqvitas 10, pp. 73-80. Priego de Córdoba.

MartínEZ CAStro, A.; Tristell MuÑoz, F. J. (2000): «Nuevos aspectos sobre los llamados «braserillos» ibéricos a la luz de un reciente descubrimiento. El conjunto de Cuesta del Espino (Córdoba)», Antiqvitas, 11-12, pp. 19-26.

Melchor GiL, E. (1990): «Comunicaciones entre Astigi y la Campiña de Córdoba en época romana: Vía Augusta y Camino de Metedores», Ariadna, 8 (1990), pp.71-97.

Melchor GiL, E. (1995): Vias romanas de la provincia de Córdoba. Córdoba.

Moltó, S.; ReIG, C. (1996): «La sepultura 53 de la necrópoli ibèrica de La Serreta»Recerques del Museu d'Alco $i \mathrm{~V}$, pp. 121-135.

Murillo Redondo, J. F. et alii (1989): «Aproximación al estudio del poblamiento ibérico en el sureste de Córdoba: unidades políticas, control del territorio y fronteras»,Fronteras,Arqueología Espacial,13, pp. 151-172.

Quesada SANZ, F. (1988): «Las acanaladuras en las hojas de falcatas ibéricas» CuPaUAM, 15, pp. $275-$ 299. Madrid.

Quesada SANZ, F. (1990): «Falcatas ibéricas con damasquinados en plata», Verdolay 2, pp. 45-59. Murcia.

QuesAdA SANZ, F.(1992): Arma y símbolo: La falcata ibérica. Instituto de Cultura Juan Gil-Albert. Alicante.

QuesadA SANZ, F. (1992): «Notas sobre el armamento ibérico de Almedinilla». A.A.C. 3, pp. 113-135. Córdoba.

Quesada SANZ, F. (1995): «Un lote de armas procedente del yacimiento de Carranza (Huéscar de Granada) y la cuestión de las vías de comunicación entre Granada y Murcia», Verdolay 7, pp. 239-250. Murcia.

Quesada SANZ, F. (1997): El armamento ibérico. Estudio tipológico, geográfico, funcional, social y simbólico de las armas en la Cultura Ibérica (siglos VI-I a. C.). Monographies instrumentum 3. Montagnac.

QUESADA SANZ, F. (2001): «Rellenando los mapas: Nuevos conjuntos funerarios ibéricos con armas en la provincia de Córdoba». Antiqvitas, 13, pp. 73-103. Priego de Córdoba.

Quesada Sanz, F.; Gabaldón Martínez, M.; Requena, F.; Zamora, M. (2000): «¿Artesanos itinerantes en el mundo ibérico? Sobre técnicas y estilos decorativos, especialistas y territorio». III Reunió sobre economía en el Mon Ibèric. Saguntum-P.L.A.V. Extra 3, pp. 291-301.

ReIG SEgui, C. (2000): «El armamento de la necrópolis ibérica de la Serreta de Alcoi (Alicante, España). Gladius XX, pp. 75-117. Madrid.

Ruiz Mata, D.; Niveau de Villedary y Mariñas, A. M.a; Vallejo Sánchez, J. I. (1998): «La ciudad tartésica-turdetana»..Actas del Congreso Internacional «Los Iberos,príncipes de Occidente. Las estructuras de poder en la sociedad ibérica». Saguntum. Extra 1, pp. 65-82. Valencia.

Ruiz Rodríguez, A.; Molinos Molinos, M. (1993): Los iberos.Análisis arqueológico de un proceso histórico. Barcelona. 
Sierra Montesinos, M. (2003): «Un lote de armas procedente de la necrópolis ibérica de Torremorana (Baena, Córdoba)», Gladius XXIII, pp. 71-110.

Sierra Montesinos, M. (2004): «Dos nuevas falcetas inéditas en la provincia de Córdoba». Antiqvitas, 16, pp. 83-88. Priego de Córdoba.

Sillières, P. (1976): «La Via Augusta de Cordoue a Cadix». Mélanges de la Casa de Velázquez, 12, pp. 27-67. Madrid.

SiLliÈRES, P. (1981): «A propos d'un nouveau milliaire de la Via Augusta, une Via Militaris en Bétique». Revue des Études Anciennes, 83, pp. 255-271.

SiLlièRES, P. (1989): «Les sources littéraires et le réseau routier de l'Hispanie Méridionale a l'époque républicaine» en GonzÁLEZ, J. (ed.): Estudios sobre Urso. Colonia Iulia Genetiva, pp. 357-365. Sevilla.

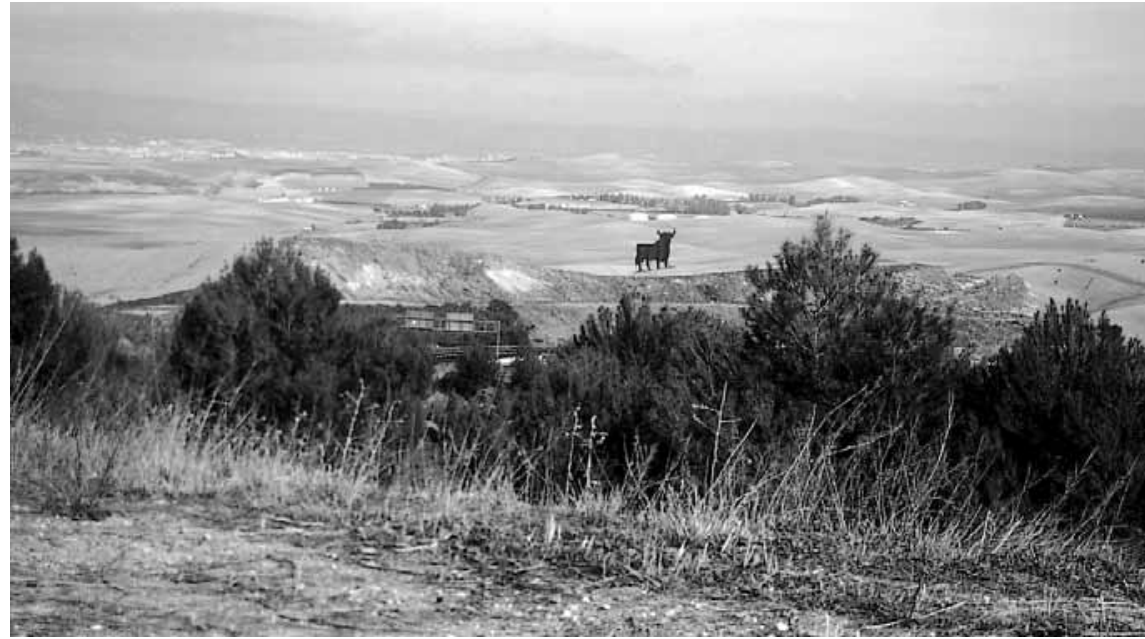

Lámina 1. Vista del yacimiento ibérico desde la zona del hallazgo.

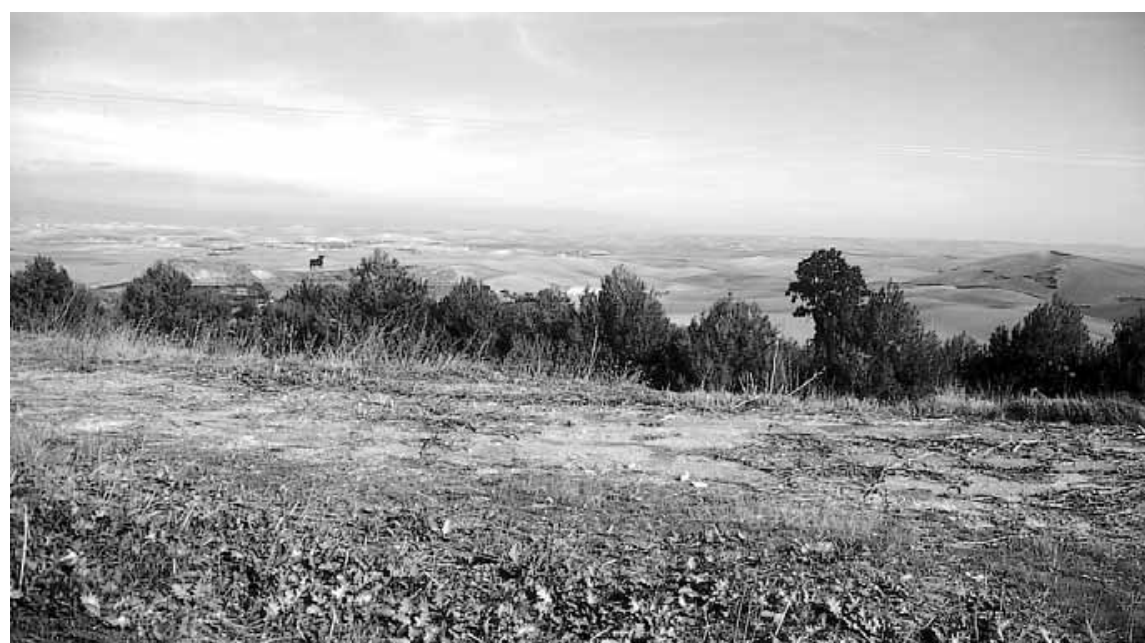

Lámina 2. Vista del amplio dominio visual desde el yacimiento, con Córdoba al fondo a la izquierda y buena parte de la campina occidental que riega el curso bajo del Guadajoz a continuación. 


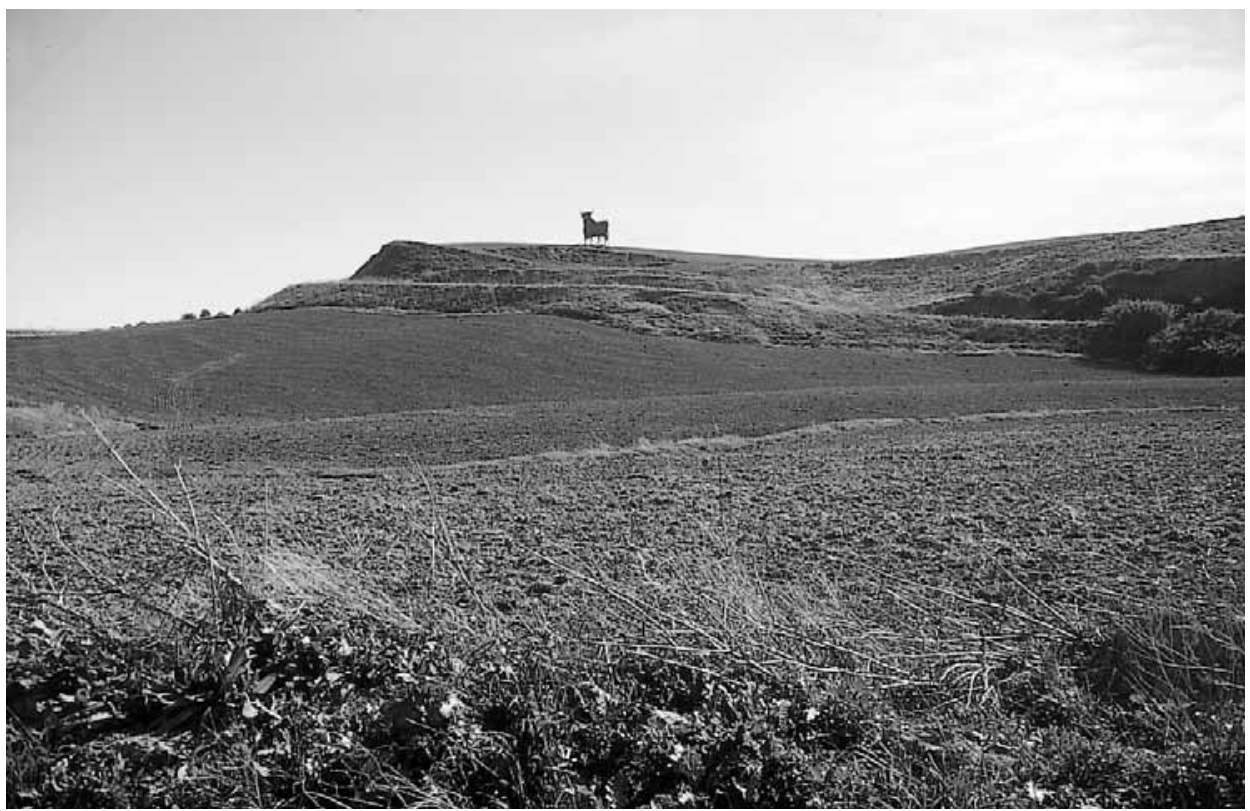

Lámina 3. Yacimiento ibérico de Cuesta del Espino desde su ladera norte.

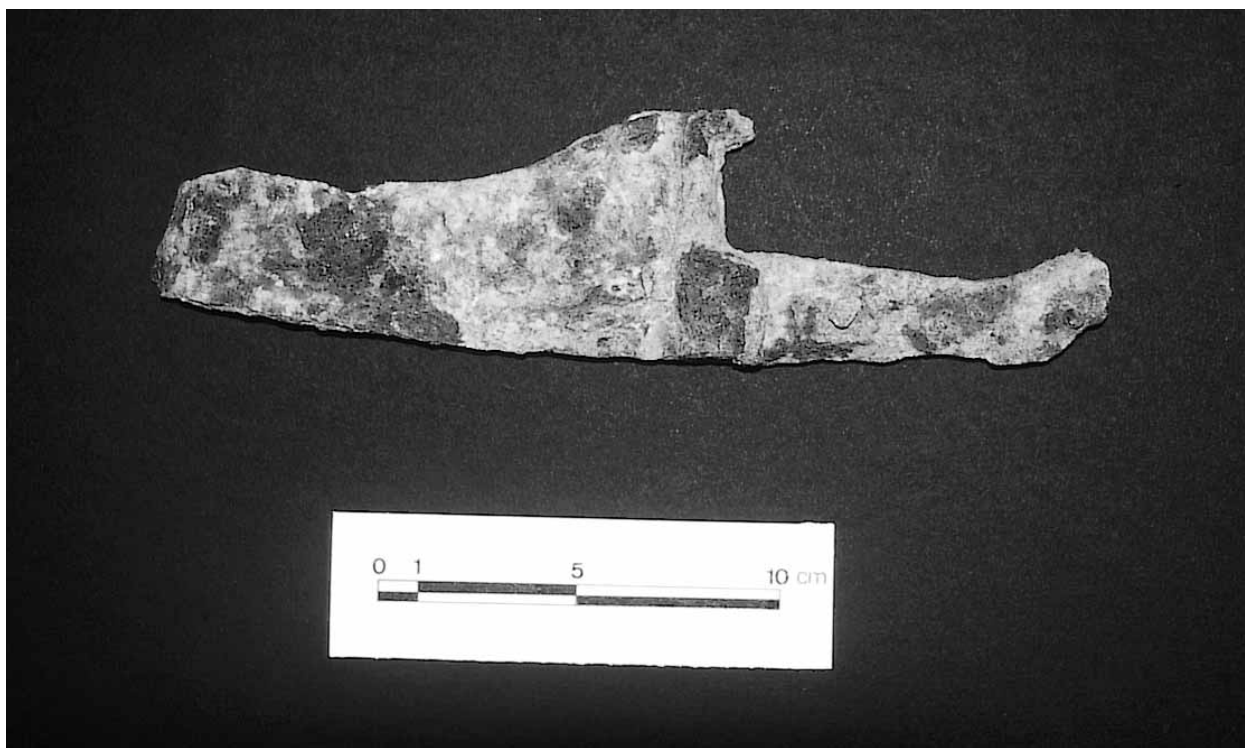

Lámina 4. Falcata del Cerro del Espino por ambas caras y el dorso. 

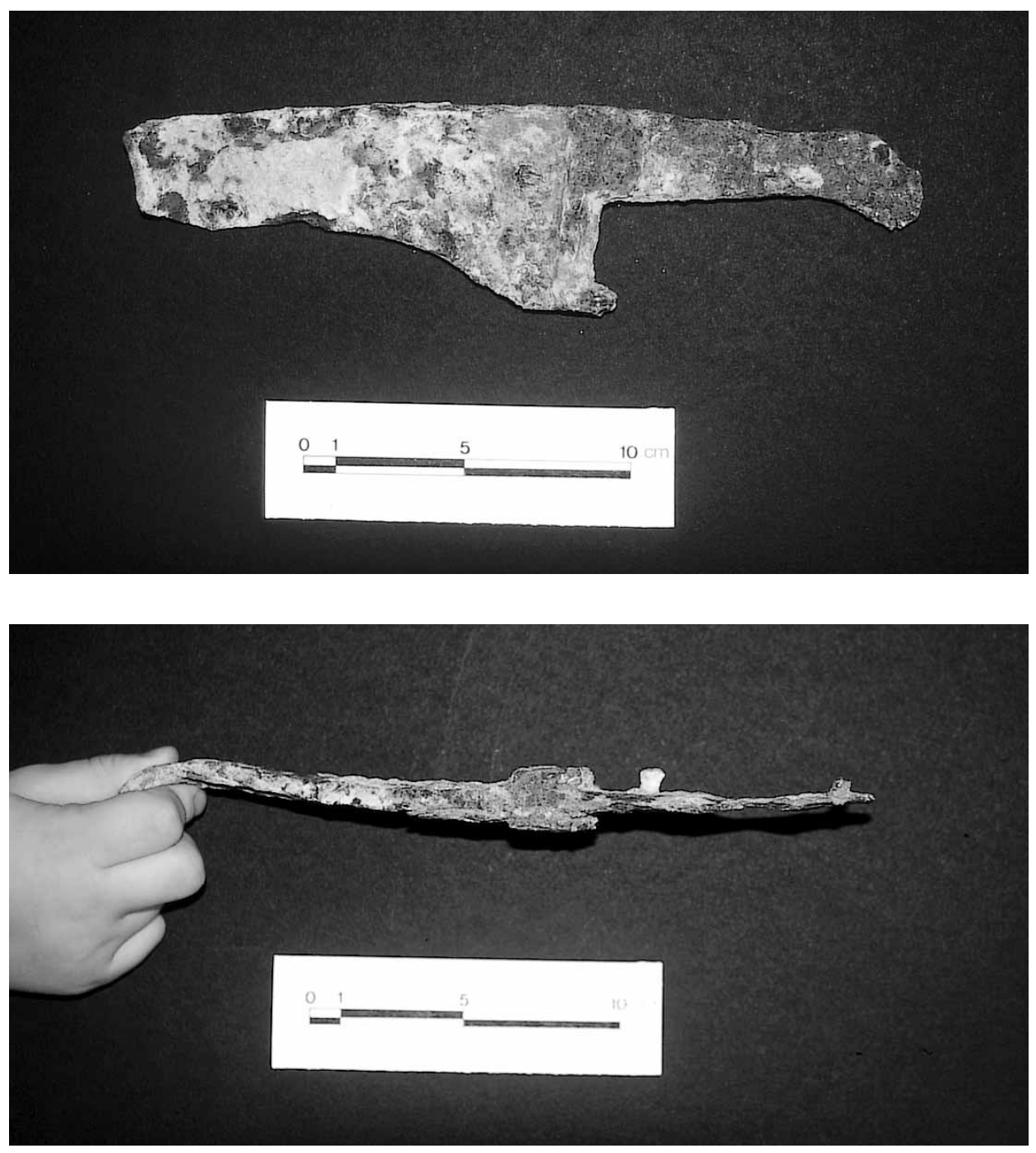

Láminas 5 y 6. Falcata del Cerro del Espino por ambas caras y el dorso.

Lámina 7: Macrofotografía de lámina de óxido desprendida de la guarda basal de la falcata donde se aprecian dos hojas de hiedra en plata.

Recibido: 07/10/2005

Aceptado: 01/04/2006

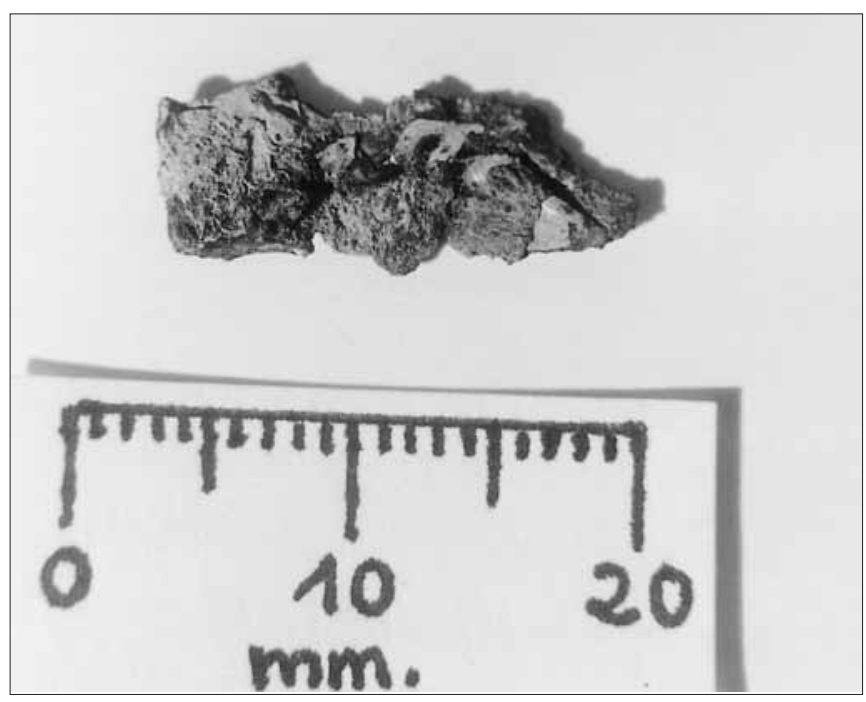

